\title{
Simulation of Comet Impact and Survivability of Organic Compounds
}

B. T. Liu, I. N. Lomov, J. G. Blank, T. H. Antoun

July 19,2007

APS Topical Conference on Shock Compression of Condensed Matter Kohala Coast, HI, United States

June 24, 2007 through June 29, 2007 
This document was prepared as an account of work sponsored by an agency of the United States Government. Neither the United States Government nor the University of California nor any of their employees, makes any warranty, express or implied, or assumes any legal liability or responsibility for the accuracy, completeness, or usefulness of any information, apparatus, product, or process disclosed, or represents that its use would not infringe privately owned rights. Reference herein to any specific commercial product, process, or service by trade name, trademark, manufacturer, or otherwise, does not necessarily constitute or imply its endorsement, recommendation, or favoring by the United States Government or the University of California. The views and opinions of authors expressed herein do not necessarily state or reflect those of the United States Government or the University of California, and shall not be used for advertising or product endorsement purposes. 
UCRL-PROC-232850

\title{
SIMULATION OF COMET IMPACT AND SURVIVABILITY OF ORGANIC COMPOUNDS
}

\author{
B. T. Liu ${ }^{1}$, I. N. Lomov ${ }^{1}$, J. G. Blank ${ }^{2}$, and T. H. Antoun ${ }^{1}$ \\ ${ }^{1}$ Earth Sciences Division, Lawrence Livermore National Laboratory, Livermore CA 94551 \\ ${ }^{2}$ SETI Institute, Mountain View CA 94043
}

\begin{abstract}
Comets have long been proposed as a potential means for the transport of complex organic compounds to early Earth. For this to be a viable mechanism, a significant fraction of organic compounds must survive the high temperatures due to impact. We have undertaken three-dimensional numerical simulations to track the thermodynamic state of a comet during oblique impacts. The comet was modeled as a $1-\mathrm{km}$ water-ice sphere impacting a basalt plane at $11.2 \mathrm{~km} / \mathrm{s}$; impact angles of $15^{\circ}$ (from horizontal), $30^{\circ}, 45^{\circ}, 65^{\circ}$, and $90^{\circ}$ (normal impact) were examined. The survival of organic cometary material, modeled as water ice for simplicity, was calculated using three criteria: (1) peak temperatures, (2) the thermodynamic phase of $\mathrm{H}_{2} \mathrm{O}$, and (3) final temperature upon isentropic unloading. For impact angles greater than or equal to $30^{\circ}$, no organic material is expected to survive the impact. For the $15^{\circ}$ impact, most of the material survives the initial impact and significant fractions $(55 \%, 25 \%$, and $44 \%$, respectively) satisfy each survival criterion at 1 second. Heating due to deceleration, in addition to shock heating, plays a role in the heating of the cometary material for nonnormal impacts. This effect is more noticeable for more oblique impacts, resulting in significant deviations from estimates using scaling of normal impacts. The deceleration heating of the material at late times requires further modeling of breakup and mixing.
\end{abstract}

Keywords: Comet impact, shock heating, 3D modeling.

PACS: $62.50 .+\mathrm{p}, 96.25$. Pq.

\section{INTRODUCTION}

Comet impacts have been proposed as a mechanism for the delivery of organic compounds to the early Earth. Several authors have attempted to address these scenarios computationally, but with few exceptions, notably Pierazzo and Melosh [1], the simulations have been two-dimensional. Advances in computational power now allow three-dimensional parameter studies to be conducted. This work examines the survivability of organic compounds during comet impacts at various degrees of obliqueness using a threedimensional shock physics code.

\section{Organic Survivability}

Some comets have been estimated to be up to $25 \%$ organic by mass [2], but successful delivery to the Earth's surface requires that organic compounds survive the high temperatures associated with impact. This work focuses on amino acids and their thermal degradation. Under ambient conditions, amino acids can decompose at relatively low temperatures $\left(<350^{\circ} \mathrm{C}\right)$, but gas gun experiments have shown that the high pressures and reduced times associated with shock loading result in significant survival of amino acids at shock temperatures up to $870 \mathrm{~K}$ [3]. 


\section{COMPUTATIONAL MODELING}

Calculations were performed using GEODYN, a Godunov-based Eulerian code with adaptive mesh refinement capabilities. This parallel code features high-order interface reconstruction algorithms and advanced thermodynamically consistent constitutive models described elsewhere [4] that incorporate many of the salient features of the dynamic response of geologic materials. GEODYN also utilizes high-pressure tabular equations of state to model the extreme conditions associated with shock loading.

\section{Problem Setup and Material Modeling}

The comet impact was modeled as a 1-km diameter sphere of ice at $200 \mathrm{~K}$ impacting a basalt half-space. The sphere impacts the surface at 11.2 $\mathrm{km} / \mathrm{s}$ (Earth's escape velocity) and begins the simulation tangent to the surface; $90^{\circ}$ (normal), $60^{\circ}, 45^{\circ}, 30^{\circ}$, and $15^{\circ}$ impacts were undertaken. Calculations were performed with a resolution of $12.5 \mathrm{~m}$ on the finest refinement level. The domain was a half-space centered on the impact point (due to the bilateral symmetry). The domain extended $12.8 \mathrm{~km}$ along the horizontal axes and $4.8 \mathrm{~km}$ and $3.2 \mathrm{~km}$ above and below the impact point, respectively. Taking advantage of the adaptive mesh refinement, the entire mesh consisted of slightly more than ten million cells.

The basalt model was developed by scaling a hard rock model calibrated to match underground explosions in granite [5]. This model includes pressure hardening, strength and failure, bulking, and porous compaction. The comet material was modeled as water; its strength was assumed to be negligible compared to basalt. Both materials were described using tabular (LEOS) equations of state to accurately capture their high pressure behavior.

\section{Survival Metrics}

In our model, the survivability of organic compounds is tied to the condensed phases of water. This most likely is a conservative estimatethat is, our calculations provide a lower boundary estimate for survival-because, under ambient pressure, organic compounds volatilize at temperatures higher than that for water.

Here, three separate metrics are used to determine the survival:
1) The peak temperature experienced by each material point. This metric does not account for kinetic or pressure effects, and thus probably underestimates the survival rate. The threshold value of $870 \mathrm{~K}$ from gas gun experiments [3] is used to indirectly account for pressure effects.

2) The thermodynamic state of the cometary material. This criterion assumes that any material that remains in a condensed phase survives the impact. Since the phase is dependent on both temperature and pressure, this implicitly takes the pressure effects into account. The comet is modeled as pure water, so this criterion is only effective up to the critical point of water $(\sim 650 \mathrm{~K})$; the phase of particular organic materials may be more relevant but involves too much uncertainty for this work.

3) The estimated final temperature on unloading. The state at the end of the simulation $(\sim 1 s)$ is unloaded isentropically to ambient pressure. The resulting state is readily mapped to existing thermal decomposition data and provides an approximate upper bound for survival at a given temperature. A very conservative threshold temperature of $373 \mathrm{~K}$ is used for this work.

\section{RESULTS AND DISCUSSION}

The simulations were run out until comet material reached the edge of the computational domain (except in the $15^{\circ}$ case, where the domain was extended). In Fig. 1, the peak temperature of each material point, mass averaged over the entire comet, is shown for the impact angles studied.

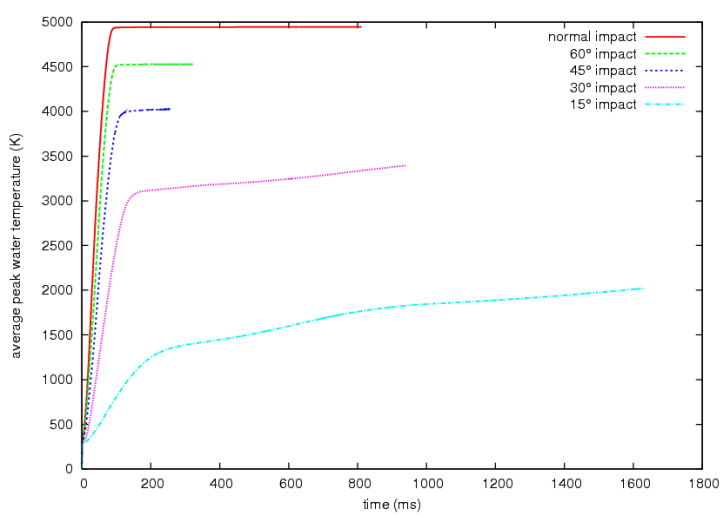

Figure 1. Peak temperatures for various impact angles. 

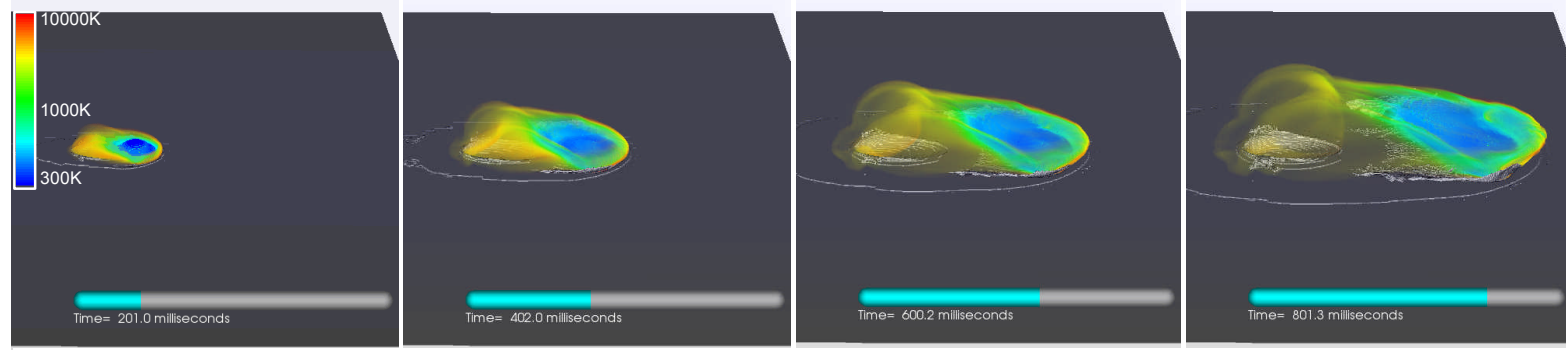

Figure 2. Volume rendering of comet temperature for $15^{\circ}$ impact. Temperature scale is logarithmic from 300 to $10,000 \mathrm{~K}$.

As expected, the peak temperatures decrease as the impact becomes more oblique. The initial rise can be attributed primarily to the heating due to shock compression of the comet. The peak temperature for the more normal impacts $\left(\geq 45^{\circ}\right)$ levels off after the initial rise, indicating that the temperature of the initial shock equal to or greater than any subsequent temperature. For the more oblique angles $\left(15^{\circ}\right.$ and $\left.30^{\circ}\right)$, however, the peak temperature continues to rise after the initial shock has passed ( 200ms). This late time heating can be attributed to the conversion of kinetic energy to internal energy as the comet decelerates after the initial impact. Figure 2 shows a volume rendering of the comet temperature for the $15^{\circ}$ impact. For this low impact angle, almost no crater is formed and very little of the material remains in the crater. Most of the material appears to "skid" along the ground, resulting in heating due to deceleration.

\section{Estimated Survivability}

Table 1 shows the fractional survival estimated using each of the three criteria. By $200 \mathrm{~ms}$ (around the time that the initial shock has passed), only the $15^{\circ}$ impact simulations shows any surviving material using the first two criteria. For the less oblique cases, the initial impact vaporizes the entire comet, heating the entire mass above the $870 \mathrm{~K}$ survival threshold. The third criterion was only applied to the impact angles of $15^{\circ}, 30^{\circ}$, and $90^{\circ}$, but again only showed survival for the most oblique case.

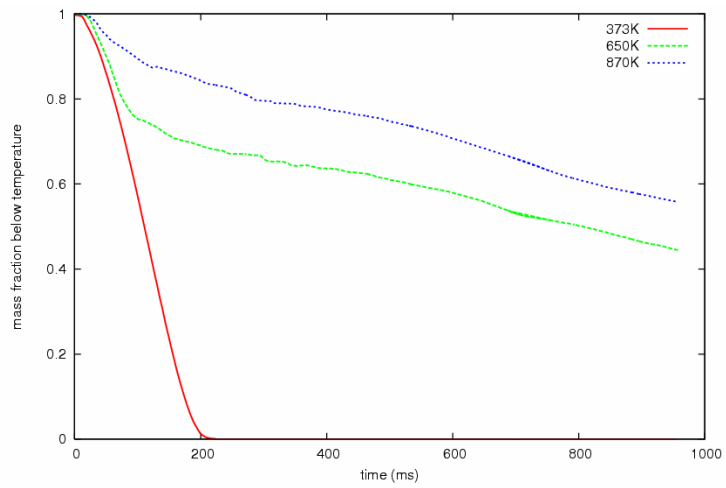

Figure 3. Fraction of material below various peak temperature thresholds for $15^{\circ}$ impact.

Figure 3 shows the fraction of material with a peak temperature below various thresholds as a

TABLE 1. Fractional survival of organic compounds estimated using various criteria.

\begin{tabular}{|c|c|c|c|c|c|}
\hline & \multicolumn{5}{|c|}{ IMPACT ANGLE } \\
\hline & $15^{\circ}$ & $30^{\circ}$ & $45^{\circ}$ & $60^{\circ}$ & $90^{\circ}$ \\
\hline \multicolumn{6}{|l|}{ Fractional Survival at $200 \mathrm{~ms}$} \\
\hline CRITERION 1: Peak temperature $<870 \mathrm{~K}$ & $82 \%$ & $0 \%$ & $0 \%$ & $0 \%$ & $0 \%$ \\
\hline CRITERION 2: Condensed phase & $75 \%$ & $0 \%$ & $0 \%$ & $0 \%$ & $0 \%$ \\
\hline Fractional Survival at end of simulation, & $1100 \mathrm{~ms}$ & $900 \mathrm{~ms}$ & $250 \mathrm{~ms}$ & $300 \mathrm{~ms}$ & $800 \mathrm{~ms}$ \\
\hline CRITERION 1: Peak temperature $<870 \mathrm{~K}$ & $55 \%$ & $0 \%$ & $0 \%$ & $0 \%$ & $0 \%$ \\
\hline CRITERION 2: Condensed phase & $25 \%$ & $0 \%$ & $0 \%$ & $0 \%$ & $0 \%$ \\
\hline CRITERION 3: Isentropic release to $1 \mathrm{~atm}, \mathrm{~T}<373 \mathrm{~K}$ & $44 \%$ & $<1 \%$ & - & - & $<1 \%$ \\
\hline
\end{tabular}


function of time for the $15^{\circ}$ impact. The initial shock heats all of the comet material above the normal boiling point of water $(373 \mathrm{~K})$. This material is not initially vaporized due to the high shock pressures. Figure 3 shows the condensed mass fraction as a function of time.

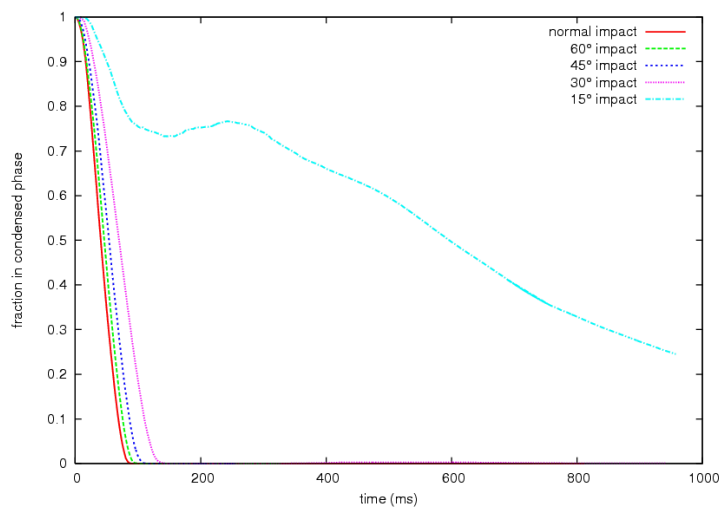

Figure 3. Mass fraction remaining in condensed phase for various impact angles.

Though a significant fraction of the material survives the $15^{\circ}$ impact to 1 second, this fraction would decrease with time due to deceleration heating. This heating will continue until the material has come to rest. However, this deceleration heating is heavily dependent on droplet formation and breakup, as well as gas phase mixing, which become important beyond one second. The relevant physics for these phenomena are not modeled in this work, but should be included to estimate final survivability.

\section{CONCLUSIONS}

High resolution three-dimensional calculations of comet impacts at various angles have been performed. For the configuration examined (1-km diameter sphere at $11.2 \mathrm{~km} / \mathrm{s}$ impacting basalt), organic materials are only expected to survive for impact angles lower than $30^{\circ}$. For oblique angles, deceleration heating was found to be important, indicating that scaling of the shock temperatures may be insufficient for low impact angles. At 1 second, a significant fraction of the material is estimated to survive a $15^{\circ}$ impact, but estimating the effect of deceleration heating at later times requires further modeling of droplet formation/breakup and gas phase mixing.

\section{ACKNOWLEDGEMENTS}

This work was performed under the auspices of the U.S. Department of Energy by the University of California, Lawrence Livermore National Laboratory under Contract W-7405-Eng-48.

\section{REFERENCES}

1. Pierazzo, E. and Melosh, H. J., "Hydrocode modling of oblique impacts: The fate of the projectile", Meteoritics and Planetary Science 35: $117-130,2000$.

2. A. H. Delsemme, A.H., "The Chemistry of Comets," Royal Society of London Philosophical Transactions Series A 325: 509-523, 1988.

3. Blank, J. G., Miller, G.H., Ahrens, M.J., and Winans, R.E., "Experimental shock chemistry of aqueous amino acid solutions and the cometary delivery of prebiotic compounds", Origins of Life and Evolution of the Biosphere 31: 15-51, 2001.

4. Rubin, M. B., Vorobiev, O. Y., and Glenn, L. A., "Mechanical and numerical modeling of a porous elastic-viscoplastic material with tensile failure," International Journal of Solids and Structures 37: 1841-1871, 2000.

5. Vorobiev, O.Y., et al, "A Strength and damage model for rock under dynamic loading", in Shock Compression of Condensed Matter, 1999 (M.D. Furnish, L.C. Chhabildas, R.S. Hixson, eds.), Volume 505, pp. 317-320. 